4 | 2017

Technologies numériques et diffusion de l'information pendant les périodes de conflits et de crise dans le monde

\title{
Les enjeux de l'utilisation du système de communication VSAT dans les processus électoraux en Afrique
}

The issues of using the VSAT communication system in electoral processes in Africa

Los retos de la utilización del sistema de comunicaciones vsat en los procesos electorales en Africa

Kondi Napo Sonhaye

\section{OpenEdition}

Édition électronique

URL : http://journals.openedition.org/ctd/738

DOI : $10.4000 /$ ctd. 738

ISSN : 2491-1437

Éditeur

Chaire Unesco Pratiques émergentes en technologies et communication pour le développement

Référence électronique

Kondi Napo Sonhaye, "Les enjeux de l'utilisation du système de communication VSAT dans les processus électoraux en Afrique », Communication, technologies et développement [En ligne], 4 | 2017, mis en ligne le 04 septembre 2017, consulté le 29 mars 2021. URL : http://journals.openedition.org/ ctd/738 ; DOI : https://doi.org/10.4000/ctd.738

Ce document a été généré automatiquement le 29 mars 2021.

Communication, technologies et développement 


\title{
Les enjeux de l'utilisation du système de communication VSAT dans les processus électoraux en Afrique
}

\author{
The issues of using the VSAT communication system in electoral processes in \\ Africa \\ Los retos de la utilización del sistema de comunicaciones vsat en los procesos \\ electorales en Africa
}

Kondi Napo Sonhaye

\section{Introduction}

1 L'instauration des régimes démocratiques dans le monde a profondément bouleversé la conception du secret, qui auparavant était lié au pouvoir. Dorénavant, c'est le peuple qui est souverain. Une obligation de «transparence politique» (Libaert, T., 2003) apparaît dans la gestion et l'organisation des États. Ainsi, le citoyen souhaite recevoir des explications, être rassuré, afin d'accorder sa confiance. Il revendique le droit à l'information. Dans les sociétés occidentales où la question de la transparence est omniprésente, la crise paraît être perpétuelle. Car plus la société est «transparente » plus on identifie des dysfonctionnements dans tous les domaines. La question de la transparence va donc de pair avec le développement des démocraties (Pascal Dauvin, 2006). Elle devient un défi en période électorale surtout en Afrique, où la démocratie est encore naissante. L'usage des technologies numériques a particulièrement été déterminant dans les mobilisations citoyennes en temps de crise en Afrique.

2 Ces technologies sont-elles efficaces? Ont-elles fait l'objet de test ou d'évaluation préalable par ailleurs avant leurs applications dans les systèmes à démocratie fragile? C'est cette problématique de transparence qui est posée dans cet article sur le prisme 
de l'utilisation du système de communication VSAT $^{1}$ et de l'évaluation de son efficacité à endiguer les crises. Le système peut- il aider à éviter ces crises ? Il nous a semblé nécessaire de nous interroger sur ces questions, de tenter une évaluation et de discuter sur l'utilisation de ce système VSAT convoité ou décrié, dans la prévention des crises électorales en Afrique avec l'étude des cas révélateurs du Togo (2010 et 2015), de la R.D. Congo (2011) et du Burkina Faso (2012).

\section{La méthode d'évaluation}

Une démarche d'évaluation nécessite au préalable une définition de l'objet ou du dispositif que l'on souhaite évaluer. Pour cela, il convient de considérer que ce dispositif technique VSAT qui fait l'objet de notre étude, se situe dans un contexte électoral plus ou moins tendu, où il se retrouve au centre des enjeux des transparences. cet article, nous nous concentrerons exclusivement sur l'évaluation du dispositif technique en rapport avec les élections au Togo, en R.D. Congo et au Burkina Faso. Il s'agira donc d'évaluer, l'utilisation et l'acceptabilité de cet objet plutôt que l'évaluation du dispositif lui-même.

En règle générale, on peut considérer qu'évaluer un dispositif technique consiste à comparer l'objet évalué à un modèle de référence afin d'en déduire des conclusions (Huart et al.,2008). Bien entendu le «modèle de référence » peut être virtuel et envisagé comme une combinaison de recommandations adaptée au domaine de l'objet à évaluer.

7 L'évaluation doit reposer sur des critères formels et explicites. Quelles sont les dimensions d'évaluation à privilégier? De nombreux critères sont mobilisables pour l'évaluation des documents numériques par exemple et dans un contexte de dispositif numérique ou technique. On distingue habituellement trois dimensions d'évaluation principales des systèmes interactifs (Senach, 1993) :

- l'utilité,

- l'utilisabilité,

- l'esthétique.

8 Tricot (Tricot et al., 2003) ajoute à ces trois dimensions l'acceptabilité (sentiment positif ou négatif à l'égard du produit, "intention » d'utilisation en fonction de nombreux paramètres : motivation, affects, culture, valeurs). C'est cette fonction qui reste sujette à discussion par les acteurs du processus électoral et qui fait l'objet de note étude. Quant aux notions d'utilité et d'utilisabilité, elles sont souvent discutées dans la littérature ainsi que le périmètre qu'elles embrassent (Huart et al., 2008). De ces trois dimensions, la plus importante semble être l'utilité.

\section{Contexte et choix des pays d'étude}

\section{Le Togo}

Depuis l'accession à l'indépendance en $1960^{2}$, le Togo a traversé plusieurs crises politiques majeures. La première en 1963 avec un coup d'Etat militaire, qui fut en Afrique le premier du genre et la seconde crise en 1967 avec l'arrivée au pouvoir du général Eyadema. Au début des années 1990, le Togo était entré dans un processus de démocratisation marqué par d'importants troubles sociopolitiques. Cette situation 
d'instabilité avait entraîné la suspension de la coopération de l'Union européenne et d'autres partenaires au développement.

Pour lever les contraintes économiques liées aux pesanteurs politiques, le Gouvernement togolais avait engagé des consultations avec l'Union européenne. Elles avaient abouti en avril 2004, à la souscription de 22 engagements pour l'instauration d'une démocratie dite apaisée au Togo, après plus d'une dizaine d'années de crise. Le processus de discussion avec tous les partenaires et la mise en œuvre de ces engagements étaient largement avancés quand intervint le décès du Président d'alors Gnassingbé Eyadema. Cet événement avait entraîné de sérieux bouleversements dans la vie sociopolitique du pays.

Figure 1 : Carte de l'Afrique de l'Ouest avec focus sur le Togo ${ }^{3}$

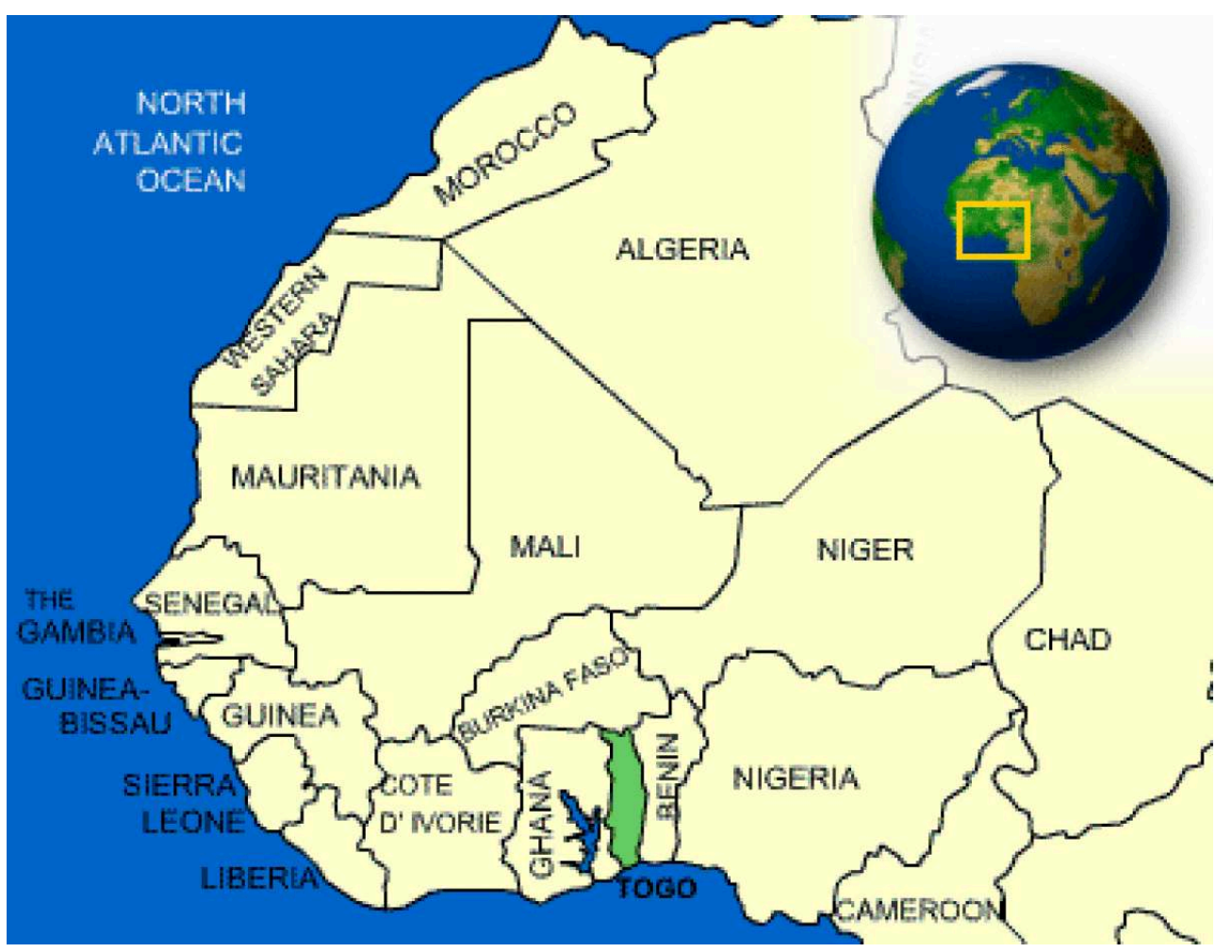

Après l'organisation de l'élection présidentielle trois mois plus tard, le 24 avril 2005, marquée par de nombreux troubles et des pertes en vies humaines, les nouvelles autorités avaient entrepris de parachever la mise en œuvre de 22 engagements pris avec l'Union européenne. Un dialogue politique s'était ainsi engagé incluant toutes les sensibilités nationales. Il avait abouti à la signature de l'Accord Politique Global (APG), le 20 août 2006 et à la formation d'un gouvernement dit d'union nationale. Sa principale mission était d'organiser des élections législatives transparentes et acceptables pour tous les Togolais afin de mettre fin à la longue crise sociopolitique.

Le paysage politique togolais est dominé par les cinq principales formations représentées au Parlement issu des élections de 2015 : l'Union pour la République (UNIR, 62 sièges) qui est le parti présidentiel ; l'Alliance nationale pour le changement (ANC, 19 sièges); le Comité d'action pour le renouveau (CAR, 6 sièges); l'Union des forces du changement (UFC, 3 sièges) et Sursaut national (1 siège). 
13 Le 25 avril 2015, Faure Gnassingbé a été réélu pour un troisième mandat de cinq ans avec 59 \% des suffrages contre $35 \%$ pour le leader de l'opposition, Jean-Pierre Fabre. Une élection qui a été contestée par l'opposition qui n'a pas reconnu les résultats. C'est ce processus que nous avons observé avec la suspension du dispositif VSAT mis en place et dont l'issue a amplifié la crise. Ce qui motive le choix du Togo, par les contestations répétées dans l'utilisation de ce système lors d'élection et la récurrence des crises qu'elle cause pourtant plébiscité par les deux partis et décrié à tort ou à raison lors de son usage.

\section{Le Burkina Faso}

14 La Haute-Volta, colonie française, accède à l'indépendance en août 1960. Son premier président, Maurice Yaméogo, crée un régime de parti unique de fait, autour de l'Union démocratique voltaïque - Rassemblement démocratique africain. Son autoritarisme et les mesures d'austérité impopulaires lui valurent l'hostilité de la chefferie coutumière et des syndicats et finalement son renversement par l'armée en janvier 1966.

Figure 2 : Carte de l'Afrique de l'Ouest avec focus sur la République du Burkina Faso ${ }^{4}$

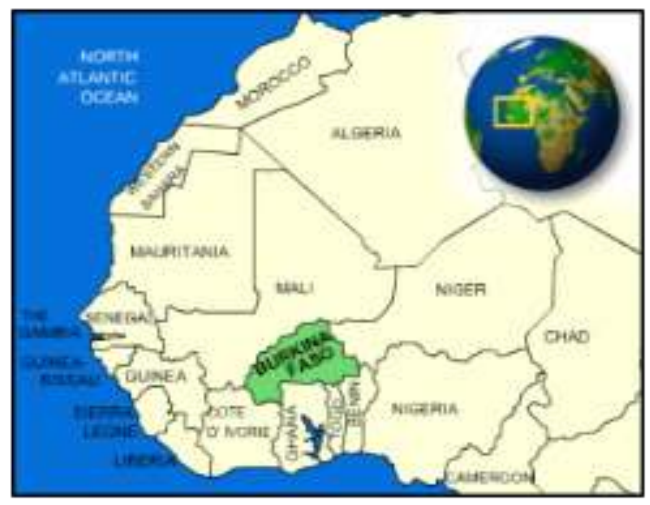

15 C'est le début de l'instabilité politique et des crises à répétition. On observera l'arrivée au pouvoir des militaires tels que successivement le lieutenant-colonel Sangoulé Lamizana, le colonel Saye Zerbo, le commandant Jean-Baptiste Ouédraogo et le capitaine Thomas Sankara, issu d'une génération plus jeune et qui, influencé par les idées d'extrême-gauche d'inspiration marxiste- léniniste, changera le nom du pays en Burkina Faso et sera remplacé à sa mort par le capitaine Blaise Compaoré qui demeurera au pouvoir pendant vingt-sept ans (1987 - 2014).

En 2014, après le départ de Blaise Compaoré, contraint à la fuite par un mouvement populaire, une charte de la transition est adoptée de manière consensuelle par les partis politiques, les organisations de la société civile, les forces de défense et de sécurité et les autorités religieuses et coutumières. Elle prévoit notamment la nomination d'un président de transition civil et l'organisation des élections présidentielles et législatives dans un délai d'un an. Le Conseil national de la transition compte des représentants des partis politiques de l'ancienne majorité et de l'opposition, des organisations de la société civile et des forces de défense et de sécurité. Les autorités de transition eurent pour mission principale d'organiser les élections. Elles doivent faire face aux tensions liées à la place du régiment de sécurité présidentielle et à la question de l'inclusivité des scrutins. En avril 2015, le Conseil 
national de la transition adopte un code électoral rendant inéligibles toutes les personnes ayant soutenu un changement anticonstitutionnel qui porte atteinte au principe de l'alternance démocratique, notamment au principe de la limitation du nombre de mandats présidentiels ayant conduit à une insurrection.

L'élection présidentielle et les élections législatives ont eu lieu le 29 novembre 2015. Roch Kaboré a été élu avec $53 \%$ des voix, contre $30 \%$ à Zéphirin Diabré. Aux législatives, le Mouvement du peuple pour le progrès obtient 55 sièges sur 127, contre 33 à l'Union pour le progrès et le changement et 18 au Congrès pour la démocratie et le progrès. L'investiture du président Roch Kaboré, le 29 décembre 2015 a mis fin à la transition.

Cette élection a été citée comme un exemple de réussite grâce d'une part à l'utilisation $\mathrm{du}$ système VSAT dans la transmission des résultats des élections présidentielles et législatives et d'autre part à une organisation consensuelle sous supervision de la communauté internationale. C'est une illustration intéressante pour cette étude.

Figure 3 : Carte de l'Afrique centrale avec focus sur la République Démocratique du Congo ${ }^{5}$

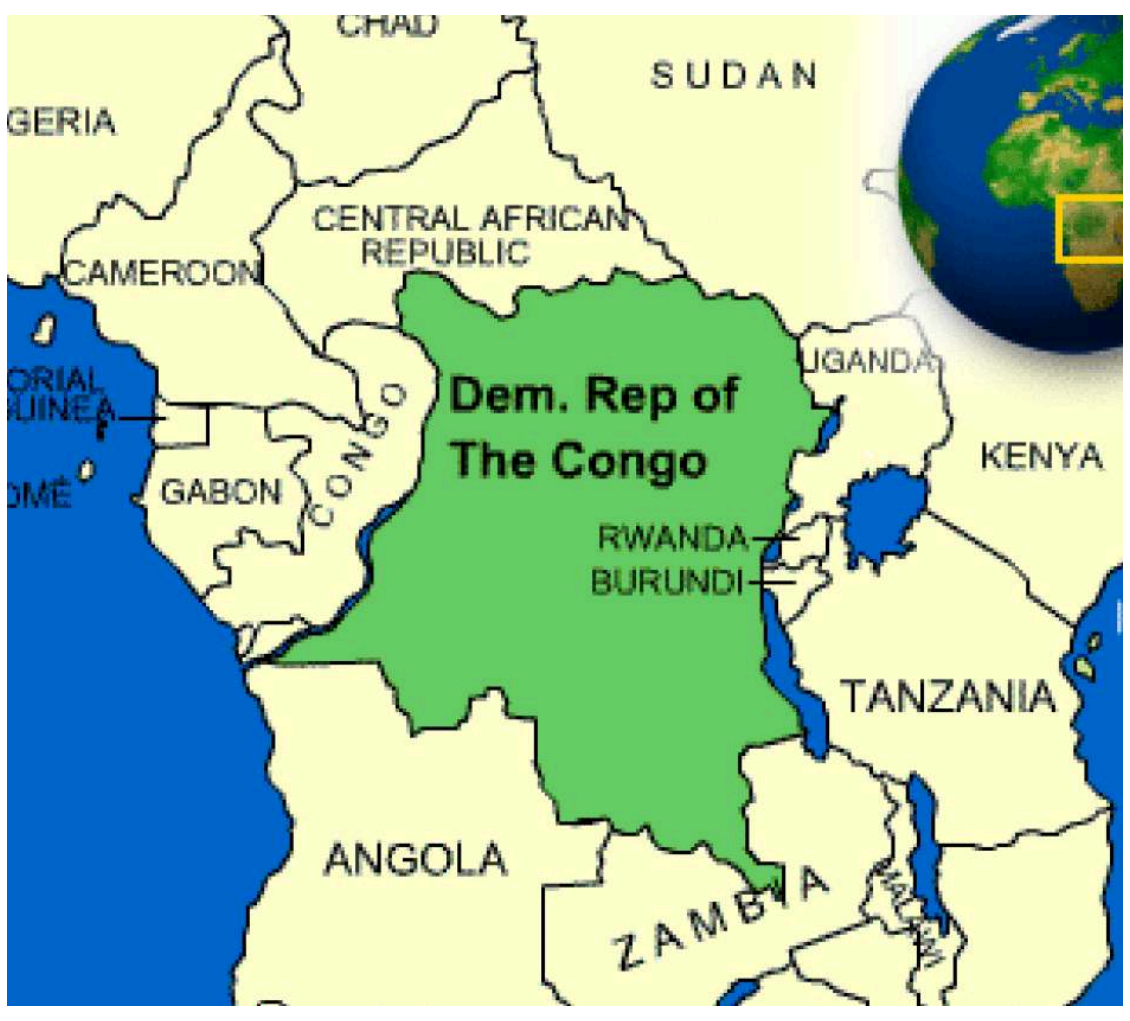

\section{La République du Congo Démocratique (RDC)}

Marqué aussi par plusieurs crises et guerres depuis son indépendance en $196^{6}$, le processus de transition mis en place après le décès de l'ancien Président Laurent Désiré $\mathrm{Kabila}^{7}, \mathrm{~s}^{\prime}$ est achevé avec l'organisation, dans de bonnes conditions, du référendum sur la Constitution de la IIIe République (18 décembre 2005) et des élections présidentielles, législatives et provinciales (scrutins des 30 juillet et 29 octobre 2006). Ces scrutins ont été un succès grâce à une participation importante du corps électoral $(65 \%$ en 
moyenne) et Joseph Kabila l'a emporté au second tour de la présidentielle face à JeanPierre Bemba avec près de $57 \%$ des voix.

Les élections présidentielles et législatives du 28 novembre 2011 s'étaient moins bien passées qu'en 2006. La campagne s'est déroulée dans un climat tendu et a été marquée par plusieurs incidents. L'opposition s'est présentée désunie face au Président Kabila. Les chefs de parti d'opposition les plus importants sont Etienne Tshisekedi (UDPS), Vital Kamerhe (UNC), Kengo Wa Dondo (UFC). Arrêté en 2008 après son inculpation par la cour pénale internationale et détenu depuis lors à La Haye, Jean-Pierre Bemba (MLC) n'a pas participé au scrutin. Les difficultés logistiques des deux scrutins se sont résorbées grâce à l'utilisation du Système VSAT et à l'appui de la mission d'observation européenne et le Centre Carter. Les problèmes particulièrement portés sur la compilation des résultats ont été pris en compte par ce système afin de garantir un maximum de transparence.

Selon les résultats proclamés par la Cour suprême de Justice, le président Kabila a obtenu $49 \%$ des suffrages, Etienne Tshisekedi $32 \%$ et Vital Kamerhe $7 \%$. Le président Kabila a prêté serment le 20 décembre 2011. Les élections législatives ont été remportées par les partis proches du président Kabila. L'opposition, dont l'UDPS est la composante principale, a plus d'une centaine de sièges. Elle a obtenu plusieurs postes au sein des commissions de l'Assemblée.

L'exemple de la RDC dans l'utilisation du VSAT est révélateur d'un pays qui, marqué par des crises, une étendue territoriale très vaste et morcelée par des guerres (c'est l'un des pays les plus vaste du continent africain $\left.{ }^{8}\right)$, a pu déployer et imposer le dispositif sous le regard des observateurs internationaux, comme système incontournable dans la transmission et la compilation des résultats pour garantir une transparence acceptable par tous les partis.

\section{Le processus de crise dans ces pays}

Une crise est un événement social ou personnel qui se caractérise par un paroxysme des souffrances, des contradictions ou des incertitudes, pouvant produire des explosions de violence ou de révolte. La crise est dans ce sens, une rupture d'équilibre. Les crises politiques en l'occurrence sont des moments de changements institutionnels. Elles reflètent souvent le déséquilibre entre les institutions et la vie réelle des individus. En période électorale, comme c'est souvent le cas, elles provoquent des manifestations, des mouvements sociaux, des émeutes voire des révoltes populaires graves.

En se référant aux travaux réalisés par Michèle Ansart - Dourlen, (2009), toute crise marque une rupture et le dévoilement de conflits souvent ignorés dans la vie individuelle ou collective. La réflexion sur la notion de crise permet de distinguer d'emblée deux de ces aspects : d'une part un état plus ou moins latent de tension, né de contradictions d'ordre politique, socio-économique ou psychique, au niveau individuel et collectif ; d'autre part la crise se manifestant comme excès, exaspération des conflits, d'où peuvent être issus des changements profonds d'ordre historique ou/ et politique. De toute façon, une crise est souvent porteuse de ruptures, elle apparaît comme « un changement brusque et décisif dans le cours d'un processus » (René Kaès, 1979). 

enjeux d'une lutte contre la terreur et la censure. De son origine étymologique grecque, la situation de crise indique l'urgence d'une prise de décision. Or, les risques encourus et la force de la propagande ne pouvaient être défiés que par un certain type de personnalité. Mais les enjeux des crises contre les régimes totalitaires et contre le déni de la rupture sont aussi d'ordre culturel. Ils peuvent être déniés par évitement ou déplacement. Sur ce point, il faut évoquer les réflexions d'Hannah Arendt (1961) sur la vérité et le mensonge en politique. Il y a impossibilité, pour des individus pénétrés des valeurs rationalistes et humanistes de vérité, de droit et de justice, à supporter l'usage systématique de faits avérés, et de mensonges d'ordre idéologique. Or, on a assisté à une généralisation de l'usage du mensonge dans les pays totalitaires. dans lesquelles s'opposent des intérêts de groupes ou de nations. Et la capacité des individus d'admettre ou de refuser les jeux d'influence et d'intérêt ne dépend pas seulement de la répression effective exercée; dans les sociétés relativement libérales du monde contemporain, les rivalités socio-économiques et les compétitions mondiales entretiennent un sentiment d'impuissance politique qui fait obstacle à l'esprit de résistance au mensonge. Il est illusoire, souligne Arendt, de croire à la suppression totale du mensonge en politique ; les mensonges d'opportunité et les «secrets d'État " sont inhérents à l'exercice du politique, serait-il essentiellement voué à l'intérêt général. Les mensonges peuvent être "des outils nécessaires et légitimes ». Mais leur usage systématique et cynique, et la passivité d'individus qui renoncent à exiger des preuves des faits avancés, révèlent une stagnation de l'esprit critique et le consentement à ce qui est perçu comme la "fatalité », le renoncement à s'interroger sur le sens réel des conflits comme le dit Michèle Ansart-Dourlen, (2009).

\section{Le VSAT, objet de crise ou solution pour la transparence électorale en Afrique}

\section{Le principe de fonctionnement du VSAT}

Avant de commencer la présentation de ce système, il faut savoir que le VSAT n'est pas une technologie normalisée, mais plutôt un concept. Pour Fleury (2003), le VSAT est un système qui repose sur le principe d'un site principal (le hub) et d'une multitude de points distants (les stations VSAT). Le hub est le point le plus important du réseau, c'est par lui que transitent toutes les données qui circulent sur le réseau. Les stations VSAT permettent de connecter un ensemble de ressources au réseau. Dans la mesure où tout est géré par le hub, les points distants ne prennent aucune décision sur le réseau ce qui a permis de réaliser des matériels relativement petits et surtout peu coûteux. Dans la plupart des cas, une antenne d'environ 1 mètre permet d'assurer un débit de plusieurs centaines de $\mathrm{Kb} / \mathrm{s}$. Une station VSAT n'est donc pas un investissement important et l'implantation d'un nouveau point dans le réseau ne demande quasiment aucune modification du réseau existant. Ainsi une nouvelle station peut être implantée en quelques heures et ne nécessite pas de gros moyens. Il suffit d'un technicien spécialisé.

VSAT est un système qui est prévu pour mettre en place des réseaux de données. Mais depuis son apparition dans les années 1980, des améliorations ont été apportées au système et les constructeurs ont réussi à augmenter considérablement le nombre 
d'applications possibles avec un réseau de ce type. Grâce à toutes ces cartes, un réseau VSAT n'est plus seulement un réseau de données, mais il peut devenir un réseau téléphonique, un réseau de diffusion vidéo. Ces différentes technologies peuvent fonctionner en même temps ce qui accroît encore la modularité du système.

Voici un exemple possible de topologie VSAT utilisant différentes fonctionnalités fournies par le système.

Figure 4 : Système de transmission VSAT

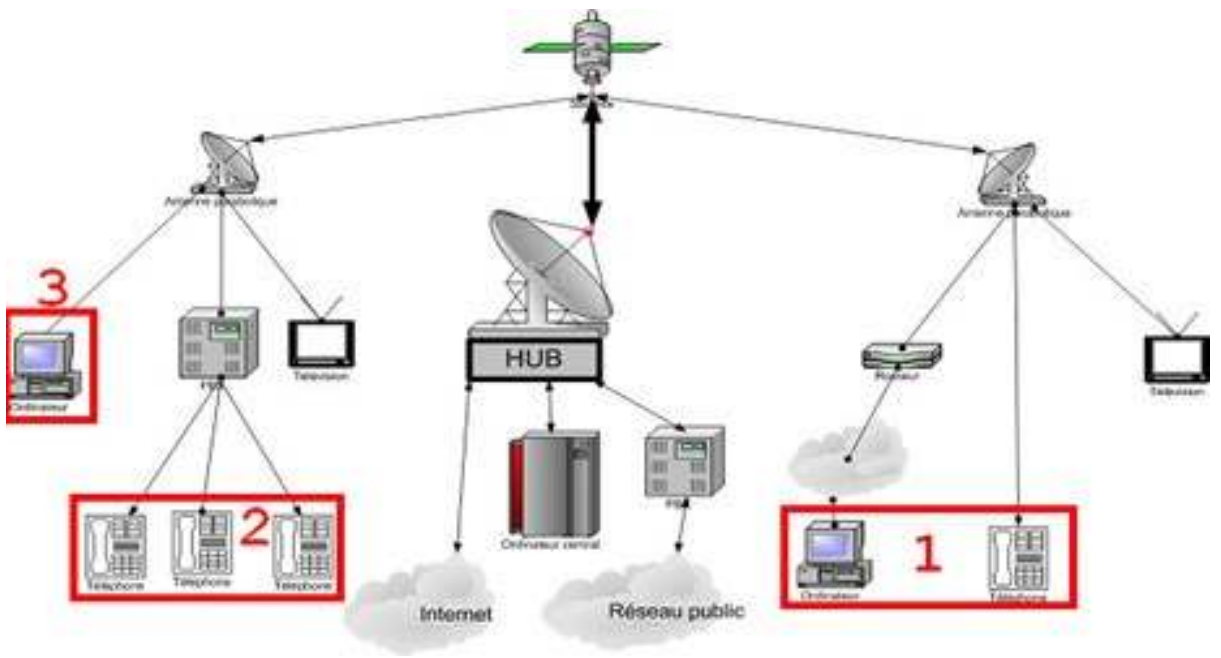

1, 2, 3 : Actions de manipulations techniques possibles

Source : Fleury (S.), GIROD (J-M.), Wantanabe (R), « Les satellites et la technologie VSAT »

La technologie VSAT permet de mettre en place des réseaux multi technologies à très grande échelle. Sa fiabilité technique ne souffre donc d'aucun doute. C'est l'un des meilleurs systèmes technologiques de transmission des données, avec toutes les garanties de sécurités. Mais dans la mesure où cette technologie a un prix relativement élevée, elle est à la base réservée aux grandes entreprises et institutions.

31 Le principal inconvénient du VSAT est son prix. Mais pour les élections, ce facteur est vite éliminé, car il est souvent pris en charge par un financement international.

Cette barrière financière relativement importante ne limite pas l'accès à la technologie.

\section{Utilisation du VSAT dans les processus électoraux}

33 La grande crainte des observateurs des élections en Afrique dans les différentes phases du processus ne sont ni les campagnes électorales même si elles peuvent être entachées de violences, ni les votes qui se déroulent souvent sans incidents majeurs. Les conflits sont observés après les scrutins lors de la restitution des suffrages exprimés. Même si, comme l'affirme Pascal Dauvin (2006), la crise ne se résume pas à l'événement visible, mais s'inscrit en amont et en aval de celui-ci. Elle s'inscrit dans une temporalité longue. (2014).

Pour revenir au système VAST ce serait donc au niveau de l'enregistrement des données ou la numérisation que des manipulations ou des biais peuvent se produire (Changement ou falsification des procès verbaux ou plus encore modification des résultats avant transmission). 
35 Au Togo, pour parer à toutes éventualités de crise lors des élections de 2010 et de 2015, des institutions de transparence pour des élections ont été mises en place, notamment la Commission Électorale Nationale Indépendante (CENI). La CENI est un service de l'État togolais, une autorité administrative indépendante qui a pour mission d'organiser et de superviser les différentes consultations électorales et les référendums. Elle dispose de prérogatives de puissance publique et jouit d'une autonomie d'organisation et de fonctionnement. Son objectif est de garantir la transparence et éviter les fraudes. Cette institution est dotée d'un équipement technique de gestion des élections permettant une rapidité de l'obtention des résultats et de lever tous les doutes que peuvent exprimer les citoyens sur la transparence des élections et pour une démocratie dite apaisée.

Grâce à la mise en place de l'outil baptisé SUCCES(Système Unifié de Collecte et de Centralisation pour les Élections et les Statistiques), de la technologie VSAT, la Commission électorale Nationale Indépendante (CENI) pourra suivre en temps réel lors des élections organisées sur toute l'étendue du territoire national, depuis Lomé la capitale où est basée la commission, la compilation des résultats et en garantir la transparence.

37 A en croire la ministre des Télécommunications en place lors des élections, l'utilisation de ce dispositif (VSAT/SUCCES) réduirait les fraudes, garantirait la transparence dans le processus électoral afin d'éviter la crise avant et après les élections. Ce qui n'était pas de l'avis de tous les partis politiques et même de certaines organisations de la société civile qui en ont rejeté son utilisation, en donnant comme argument son «manque de transparence » et ses possibles manipulations. Un responsable politique de l'opposition et membre de la CENI, donnait au lendemain des élections, les raisons pour lesquelles l'opposition rejette « SUCCES ».

Intervenant sur une radio locale, il a donné quatre raisons pour lesquelles il ne peut avoir confiance en ce système. Selon lui, la transmission des résultats par voie électronique a été expérimentée en 2010 à travers l'utilisation du VSAT. «Lorsqu'on a commencé à proclamer les résultats à la CENI et qu'on avait constaté qu'un candidat était en avance, on nous a dit que le VSAT était tombé en panne. Selon l'expert sénégalais, le système avait été bloqué. Après, les résultats ont été manipulés ", avait-il déclaré.

Autre raison avancée, « en 2013, les résultats étaient publiés au moment même où les résultats sortis des urnes n'avaient pas atteint les Commissions Électorales Locales Indépendantes (CELI). Sans plénière de la CENI, la tendance des résultats est devenue résultats alors que la CENI n'avait pas validé ces résultats", a-t-il regretté. Cette manière de publier les résultats, a-t-il souligné, consiste à mettre les Togolais devant le fait accompli avant que la CENI ne rassemble les véritables résultats sortis des urnes. « Nous ne l'accepterons pas ", prévientil.

Pour lui, la loi électorale n'a pas prévu cette procédure électronique, mais plutôt le comptage des votes à partir des procès-verbaux. C'est ce vide juridique qui peut aussi être évoqué pour bloquer le processus.

«La loi électorale donne des indications précises. Le moyen pour la transmission des résultats, c'est les procès-verbaux, les feuilles de dépouillement élaborés et conçus dans les bureaux de vote, la fiche d'émargement des électeurs. C'est à partir de ces éléments que la Cour constitutionnelle peut donner ses appréciations sur le contentieux électoral. Nous avons été surpris aujourd'hui qu'on associe à cette procédure évoquée par la loi électorale en ses articles 102 et 103 une autre 
procédure qui est une procédure électronique non prévue par la loi », a indiqué ce responsable 9 .

41 Selon ce membre de la CENI, la gestion du système de proclamation des résultats par voie électronique se fait parallèlement à la CENI. L'opposition redoute également la présence à la CENI d'un expert étranger connu pour des actes de manipulation. «Il est le cerveau de SUCCES. C'est un expert en mission pour le pouvoir ", avait-il laissé entendre.

«Nous ne voulons pas qu'après les élections qu'il y ait encore des morts, qu'on proclame quelqu'un qui n'a pas gagné qu'il est le vainqueur. Nous voulons que cette fois-ci les Togolais soient rassurés que le vainqueur de cette élection soit celui qui a été choisi par eux. Nous n'allons pas continuer par proclamer de faux résultats dans ce pays ", concluait-il.

Ces déclarations rendues publiques en son temps pouvaient être retrouvées dans les journaux.

43 Après ces remises en cause de l'opposition, la commission électorale togolaise avait annoncé les difficultés de l'utilisation du système de communication (VSAT/SUCCES) pour la centralisation des résultats de la présidentielle en évoquant des «difficultés techniques ». D'après le président de la commission électorale, le système VSAT de communication par satellite rencontre des problèmes techniques. Il explique vouloir voir le décompte physique des voix afin d'établir les résultats du scrutin. SUCCES a été donc abandonné.

Quant à l'opposition, elle affirme ne plus faire confiance aux résultats envoyés par VSAT, pourtant préalablement considéré comme un système difficile à pirater.

Le candidat du principal parti d'opposition avait accusé le parti au pouvoir de tenter de manipuler les résultats du scrutin avec ce système VSAT.

Ancienne colonie française, le Togo n'a jamais connu d'élection libre et équitable depuis son indépendance, dans les années 1960. En 2010, le système VSAT faisait l'unanimité pour tous les partis et sa non-utilisation, considérée par l'opinion comme un manque de transparence. A l'époque, dans un communiqué rendu public lors du dépouillement des votes le président de Synergie Togo, une association de la société civile avait rappelé les conditions qui devaient être requises pour des élections transparentes et acceptables pour tous les partis. Selon lui, sur le « mieux voter », étape préparatoire des élections, il est à signaler le refus d'utilisation du système VSAT garantissant une transmission fiable des résultats des centres électoraux locaux vers le centre national, au profit d'un système parallèle installé la veille du scrutin par la Commission Electorale Nationale Indépendante (CENI).

Sur le «Vrai restituer", selon ses termes, la restitution de la vérité des urnes dans le système de transmission des résultats devrait passer par voie directe par les CELI, au lieu de passer par la transmission VSAT. Les résultats n'ont donc pas pu être contrôlables.

Pour lui compte tenu de tous ces éléments concordants, il apparaît que le processus électoral a délibérément manqué de transparence, de justice et de fiabilité, ce qui en entache gravement sa crédibilité, tant au regard des normes internationales que des moyens humains et financiers engagés par l'Union européenne.

Synergie-Togo ne peut soutenir des résultats sortis des urnes avec autant d'électeurs fictifs et d'irrégularités. La validation de ces résultats n'est pas de nature à ramener la paix dans un pays dont les lendemains restent incertains compte tenu de l'histoire 
électorale récente du pays, avec plus de 500 morts lors des élections présidentielles de 2005.

50 L'enquête que nous avons réalisée au cours des élections de 2015, dénote quelques réserves face à l'utilisation de ce système. Les membres de la CENI, étaient très départagés par rapport à ce "système ». De plus certains électeurs et acteurs politiques n'étaient pas favorables à son usage, à cause du manque de confiance qu'ils avaient dans les personnes à qui la responsabilité de manipulation (enregistrement des données ou la numérisation des procès verbaux ou des résultats avant transmission) avait été confiée ou conférée.

51 Il est clair que le dépouillement manuel arrangeait plus les partis acceptant le VSAT (partie gouvernementale) que l'opposition. La question qui peut se poser est de savoir si les partis d'opposition qui au dernier moment avaient refusé l'utilisation du matériel VSAT, n'étaient pas tombés dans le piège du pouvoir ? Question difficile à répondre tant les manipulations d'opinion étaient observées des deux côtés.

52 Les deux autres cas où le VSAT a été utilisé avec des résultats sans contestation sont le Burkina Faso et la R.D. Congo comme nous l'avons évoqué précédemment.

53 Au Burkina Faso, lors des élections législatives et municipales de 2013 organisées avec l'appui du Programme des Nations Unies pour le Développement (PNUD) et des autres partenaires au développement, en vue d'apporter un appui à la CENI pour l'organisation d'élections libres, démocratiques, transparentes et inclusives, le VSAT a été utilisé comment système moteur de transparence. Cette assistance technique et financière, coordonnée par le PNUD à travers le mécanisme du "Panier commun", s'inscrit dans le cadre d'un processus électoral crédible et pérenne. Et 2016, la commission d'organisation des élections s'est inspiré des ces élections pour organiser un scrutin transparent, l'un des premiers non contestés dans ce pays.

54 Le cas de la République Démocratique du Congo, dans l'utilisation du système VSAT pour les élections sans crise en 2011 a été aussi intéressant dans notre étude par l'implication active des acteurs indépendants du processus. L'Organisation des Nations Unies avec l'appui des observateurs du Centre Carter ont été les initiateurs de ces élections au Congo Démocratique. C'était en son temps, l'une des rares élections non contestées dans le pays et qui avait ramené la paix après une dizaine d'années de crises et de guerres.

55 Le matériel de transmission électronique (VSAT) était présent dans $73 \%$ des cas observés, mais les observateurs n'ont pas pu confirmer si ce matériel était effectivement opérationnel et si son utilisation était cohérente selon le rapport final ${ }^{10}$. A la fin des opérations de vote, le système a été mis en avant pour servir de preuve et valider la transparence de ces élections.

\section{Conclusion}

Il est indéniable que la communication joue un rôle crucial, en situation de crise, pour les organisations et les acteurs qu'elle soit publique ou privée. La communication est sollicitée pour informer, transmettre, rassurer la population, et rétablir la confiance. Mais une crise peut être créée par la communication elle-même. Lorsque les stratégies de communication ne sont pas partagées par tous les partenaires. De nombreuses évolutions sont constatées depuis l'émergence de la communication en période de crise 
dans les années 1980, jusqu'alors. Les différents épisodes de cette forme particulière de communication ont montré que les pouvoirs publics ont compris les enjeux et le rôle liés au dispositif qui est utilisé.

Selon McLuhan (1968), «Les matériaux sur lesquels les mots sont inscrits importent davantage que les mots eux-mêmes ». Le dispositif, exerce autant, sinon plus d'influence sur l'individu que le contenu. D'après lui, le dispositif manipulable par l'homme transforme notre façon de percevoir l'information. Le système devient une extension de l'individu. Ainsi son efficacité ne suffit plus pour être acceptable.

Mais l'effort technologique peut être à la mesure de la fragilité du système. L'évaluation dans les cas étudiés montre à quel point un dispositif peut rendre ou créer une crise complexe et difficile à gérer, lorsqu'il est installé. Il reste insuffisant pour l'instauration d'un climat de confiance mutuelle entre les acteurs. Le système peut aider à éviter les crises, s'il est établi sur des bases claires, sur l'utilisation consensuelle (acceptabilité) de ce système et sur sa transparence.

La transparence est l'une des principales exigences des citoyens à l'égard des responsables politiques et des acteurs. Le défi pour ceux-ci est de trouver l'équilibre entre ce qui doit être dévoilé et ce qui ne doit ou ne peut l'être. Pour le citoyen, la difficulté est de savoir si l'information qu'il reçoit est réellement sincère et correspond à la réalité.

60 La transparence permet au regard de traverser l'objet, mais ne l'arrête pas. A partir de ce principe élémentaire de physique, Libaert (2003), décrit dans un essai clair et concis, l'une des utopies contemporaines. Il prend le contre-pied de cette découverte d'une vertu salvatrice et affirme, au contraire, que la transparence est la pierre angulaire de la démocratie. Comme le disait Pascal Dauvin (2006), la question de la transparence va de pair avec le développement des démocraties. Dans le cas de l'utilisation d'un système de communication dans un processus électoral, certaines précautions doivent être prises afin d'éviter les crises de confiance trop récurrentes. Notre contribution permet de relever la nécessité d'établir des bases de traçabilité de la transmission de l'information afin de pouvoir reconstituer en cas de doute son itinéraire. La dématérialisation offre de grandes facilités dans la transmission des données. Elle doit aussi permettre une matérialisation et une authentification manuelle ou matérielle pour les besoins de la cause.

\section{BIBLIOGRAPHIE}

Ansart Dourlen, «La notion de crise politique et les modalités de son déni dans les mouvements contestataires et révolutionnaires. ", Les cahiers psychologie politique, $n^{\circ} 14$, janvier 2009. [en ligne], URL : http://lodel.irevues.inist.fr/cahierspsychologiepolitique/index.php?id=353, consulté le $11 / 06 / 2017$.

Laurence Favier, «L'anonymat en contexte dématérialisé : l'originalité du vote électronique », Terminal, $\mathrm{N}^{\circ} 105,1995, \mathrm{p} 27-41$. 
Sébastien Fleury, Jean-Marc Girod, Ryo Wantanabe, « Les satellites et la technologie VSAT ». Ingénieur 2000, 2003.

René Kaes, Crise et dépassement, Dunod, Paris, 1979.

Patrick Lagadec, La gestion des crises, Outils de réflexion à l'usage des décideurs, Ediscience, Paris, 1991.

Patrick Lagadec, « Pourquoi et comment bâtir un plan de communication préventive », La communication de crise, 1991, p. 81-103.

Patrick Lagadec, Apprendre à gérer les crises, Les éditions d'organisation, Paris, 1994.

Roland Laulheret, " Maîtrise des risques techniques ", Cours de technologies spatiales, Toulouse éditions Cepadues, 1998, p. 320-362.

Jean-Michel Ledjou \& Hanitra Randrianasolo-Rakotobe, (dir.), Des réseaux et des hommes. Les Suds à l'heure des technologies de l'information et de la communication - internet une chance pour l'Afrique, Karthala, Paris 2012.

Thierry Libaert, La communication de crise, Dunod-Topos, 3e édition 2010.

Thierry Libaert, La transparence en trompe-l'œil, Éditions Descartes et Cie, Paris, 2003. Edgar Morin, «Pour une crisologie », Communications, vol. 25, 1976, p. 149-163.

Roux-duf Ort, "Apprendre des crises. Entre le statu quo et la transformation », Sciences de la société, n 44, 1998, p. 165-182. Alain Pages, Michel Gondran, Fiabilité des systèmes, Edition Eyrolles, Paris, 1980.

E. Plottu, «Les crises et leur gestion », Sciences de la société, n 44, 1998, p. 145-164.

Rapport final : « Election présidentielle mars 2010 au Togo » Union européenne mission d'observation électorale, 2010.

Rapport final : « Élections présidentielle et législatives République Démocratique du Congo », The Carter Center, 28 novembre 2011.

Rapport final : « Processus électoral au Burkina Faso : session de capitalisation des acquis des élections législatives et municipales couplées du 2 déc. $2012 », 2012$.

Kondi Napo Sonhaye, Le développement de l'administration électronique en Afrique : Réflexion sur les indicateurs de comparaison internationale à partir de l'exemple du Togo, Thèse de Doctorat, Université Lille 3, 2015.

Farid Yakoubi, La communication publique de crise, Collection Risques et Crises. NHESJ, Paris, 2014. André Vitalis, (dir.), Médias et nouvelles technologies de communication. Pour une sociopolitique des usages. Éditions Apogée, Rennes, 1994.

Pierre Zemor, « Les crises collectives au XXIe siècle. Quel constat ? Quelles réponses ? ", Cahiers de la Sécurité, septembre-décembre nº 10, 2009.

Kondi Napo Sonhaye, Le développement de l'administration électronique en Afrique : Réflexion sur les indicateurs de comparaison internationale à partir de l'exemple du Togo, Thèse de Doctorat, Université Lille 3, 2015.

Farid Yakoubi, La communication publique de crise, Collection Risques et Crises. NHESJ, Paris, 2014.

André Vitalis, (dir.), Médias et nouvelles technologies de communication. Pour une sociopolitique des usages. Éditions Apogée, Rennes, 1994. 
Pierre Zemor, « Les crises collectives au XXIe siècle. Quel constat ? Quelles réponses ? ", Cahiers de la Sécurité, septembre-décembre $n^{\circ}$ 10, 2009.

\section{NOTES}

1. Very Small Aperture Terminal ou Terminal à très petite ouverture.

2. Le Togo a eu son indépendance, le 27 avril 1960, avec l'accord de l'administration française, sous le contrôle de l'ONU.

3. http://www.countryreports.org/country/Togo.htm [consulté le 03/01/2017]

4. http://www.countryreports.org/country/BurkinaFaso.htm [consulté le 03/01/2017]

5. http://www.countryreports.org/country/DemocraticRepublicoftheCongo.htm [consulté le 03/01/2017]

6. Le 30 juin 1960 l'indépendance du Congo belge est proclamée en tant que «République du Congo ", Joseph Kasa-Vubu, Président ; Lumumba Premier ministre.

7. Le 17 mai 1997, les troupes de Kabila entrent dans Kinshasa sans rencontrer de résistance. Laurent-Désiré Kabila, depuis Lubumbashi, se déclare président du pays, qu'il rebatise République démocratique du Congo. Mobutu, malade, trouve refuge à Gbadolite pour s'exiler ensuite au Maroc, où il décèdera en septembre à Rabat.

8. La République démocratique du Congo s'étend de l'océan Atlantique au plateau de l'Est et correspond à la majeure partie du bassin du fleuve Congo. Grand comme quatre fois la France, quatre-vingt fois la Belgique, une fois et demie plus grand que le Québec (Canada), ou encore grand comme la partie des États-Unis située à l'est du Mississippi, c'est le 11e État du monde par sa taille avec ses $2345409 \mathrm{~km} 2$.

9. Sources : http://afreepress.info [consulté le 02/01/2017]

10. Rapport final : «Élections présiden- tielle et législatives République Démocratique du Congo » - 28 novembre 2011 - Rapport Final - The Carter Center.

\section{RÉSUMÉS}

En démocratie, tout citoyen a un réel besoin de transparence. Il revendique à chaque moment où il est sollicité un droit à la vérité et pendant les élections, le droit à la " vérité ». Dans les sociétés occidentales où la question de la transparence est omniprésente, les crises de confiance paraissent être perpétuelles. Plus une société est «des élections ont été mises en place transparente " plus on identifie des dysfonctionnements. La question de la transparence va donc de pair avec le développement des démocraties. (Pascal Dauvin, 2014).

En Afrique où la démocratie est encore naissante, la transparence est un enjeu et un défi surtout en période électorale. Compte tenu des difficultés de terrain et de communication dans certaines zones du continent, l'usage des technologies numériques est souvent déterminant dans les mobilisations citoyennes en temps de crise. Le système VSAT est souvent sollicité après un scrutin pour la transmission et le « transport » rapide des données et des informations.

Au Togo, lors des élections de 2010 et de 2015, des institutions de transparence pour des élections ont été mises en place, notamment la Commission Électorale Nationale Indépendante (CENI). Les entretiens et enquêtes menés auprès des électeurs et des principaux acteurs de la vie politique et 
l'analyse des discours de campagne nous a permis de constater que la préoccupation première de chacun pour endiguer la crise électorale récurrente est la mise en place d'un système consensuel pouvant limiter les fraudes et garantir la transparence. C'est dans ce sens que le VSAT a été coopté par la CENI comme matériel de communication dans les deux processus électoraux sans être véritablement utilisé à cause des questions autres que le fonctionnement du système en luimême.

Les deux autres cas analysés où le système VSAT a été utilisé avec des résultats sans contestation sont ceux du Burkina Faso et de la République Démocratique (RD) du Congo.

$\mathrm{Au}$ Burkina Faso, lors des élections législatives et municipales de 2013, organisées avec l'appui du Programme des Nations Unies pour le Développement (PNUD) et des partenaires au développement ainsi que celles de 2016, le système VSAT avait été utilisé afin de garantir un scrutin transparent et sans contestation dans ce pays. Ce qui a été le cas.

En République Démocratique du Congo, vu l'immensité du pays, le système VSAT a été véritablement au cœur des élections sans crise en 2011 avec l'implication active des acteurs indépendants au processus tels que l'Organisation des Nations Unies et le Centre Carter. Cette élection avait pour objectif de ramener la paix après une dizaine d'années de crises et de guerres. Notre contribution a pour objet, les enjeux de transparence autour de l'utilisation de ce système VSAT convoité ou décrié, dans la prévention des crises électorales en Afrique.

In a democracy, Citizen seek for transparency. Notably during the election period have the citizens claimed for the right to get the truth. In Occidental societies where the issue of transparency is omnipresent, confidence crises seem to be perpetual. The more a society is "transparent" more identifying malfunctions. The transparency issue goes by pair with development of democracies. (Pascal Dauvin, 2014).

In Africa, where the democracy is at early stage, transparency is an issue and a challenge especially at election times. Given the difficulties of land and communications in some areas of the continent, the use of digital technologies is often decisive in civic mobilization in times of crisis. VSAT is often sought after voting for the transmission and the "transport" fast data and information.

In Togo, during the 2010 and 2015 elections, transparency of institutions for elections has been implemented, including the Independent National Electoral Commission (INEC). Interviews and surveys conducted among voters and key actors from politics and analysis of campaign speech allowed us to see that the first concern of everyone to curb the recurrent election crisis is the establishment of a system consensus can limit fraud and ensure transparency. It is in this sense that the VSAT was appointed by INEC as communications equipment in both electoral processes without really being used because of issues other than the operation of the system itself. The other two cases analyzed where the VSAT system was useful with results without dispute are those of Burkina Faso and the Democratic Republic (D.R.) of Congo.

In Burkina Faso, in parliamentary and municipal elections in 2013, organized with the United Nations Development Programme (UNDP) and development partners and those of 2016, the VSAT system was used to ensure ballot transparent and without challenge in this country. This has been the case.

Democratic Republic of Congo, given the vastness of the country, the VSAT system was truly at the heart of the 2011 crisis without elections with the active involvement of independent actors in processes such as the United Nations and the Carter Center. This election was intended to bring peace after a decade of crises and wars.

Our contribution is to the issues of transparency around the use of the VSAT system coveted or maligned in preventing electoral crises in Africa.

En una democracia, todos los ciudadanos tienen una necesidad real de transparencia. Se reivindica en cada momento que es solicitado, un derecho a la verdad y durante las elecciones, el 
derecho a la "verdad". En las sociedades occidentales, donde la cuestión de la transparencia es omnipresente, la crisis de confianza parecen ser perpetua. Cuanto más una sociedad es "transparente", mayor es la identificación de problemas en el funcionamiento. La cuestión de la transparencia va de la mano con el desarrollo de las democracias. (Pascal Dauvin, 2014). En África, donde la democracia es todavía incipiente, la transparencia es un problema y un reto, especialmente en época de elecciones. Dadas las dificultades de comunicación terrestre en algunas zonas del continente, el uso de la tecnología digital es a menudo decisivo en la movilización de los ciudadanos en tiempos de crisis. El sistema VSAT es hecha a menudo después de un sondeo para la transmisión de datos y rápidos de "transporte" e información.

En Togo, durante las elecciones de 2010 y 2015, la transparencia de las instituciones para las elecciones se han implementado, incluyendo la Comisión Electoral Nacional Independiente (CENI). Entrevistas y encuestas realizadas entre los votantes y los actores clave de la política y el análisis de discurso de campaña nos ha permitido observar que la primera preocupación de todo el mundo para frenar la crisis de la elección recurrente es el establecimiento de un sistema de consenso que puede limitar el fraude y garantizar la transparencia. Es en este sentido que el VSAT fue nombrado por el INEC como material de comunicaciones en ambos procesos electorales sin ser realmente utilizado debido a los problemas no relacionados con el propio sistema operativo.

Los otros dos casos analizados donde el sistema VSAT se ha utilizado con resultados sin discusión son los de Burkina Faso y la República Democrática (DR) del Congo.

En Burkina Faso, en las elecciones legislativas y municipales de 2013, organizado con el Programa de las Naciones Unidas de Apoyo para el Desarrollo (PNUD) y asociados para el desarrollo, así como los de 2016, se utilizó el sistema VSAT para asegurar y elección transparente y sin oposición en el país. Lo que ha sido el caso.

En la República Democrática del Congo, dada la gran extensión del país, el sistema VSAT estuvo verdaderamente en el centro de la crisis sin elecciones en 2011 con la participación activa de agentes independientes en procesos tales como las Naciones Unidas y el Centro Carter. Esta elección fue pensada para llevar la paz después de una década de crisis y guerras.

Nuestra contribución tiene por objetivo los desafíos de transparencia en torno al uso del sistema VSAT o codiciado difamado en la prevención de las crisis electorales en África.

\section{INDEX}

Mots-clés : évaluation, VSAT, communication, élections, crise, transparence, Afrique

Keywords : evaluation, VSAT, communication, polling, elections, crisis, transparency, Africa

Palabras claves : VSAT de comunicaciones, elecciones, crisis, transparencia, Africa

\section{AUTEUR}

\section{KONDI NAPO SONHAYE}

Université de Lomé, Togo 